\title{
Mind the gap (between assessing risks and prioritizing management)
}

\author{
Stefano Canessa', Amanda E. Trask', John G. Ewen' \\ I Institute of Zoology, Zoological Society London, Regent's Park, London, UK \\ Corresponding author: Stefano Canessa (science@canessas.com)
}

Academic editor: S. Kumschick | Received 20 November 2020 | Accepted 5 January 2021 | Published 3 August 2021

Citation: Canessa S, Trask AE, Ewen JG (2021) Mind the gap (between assessing risks and prioritizing management). NeoBiota 68: 1-4. https://doi.org/10.3897/neobiota.68.60816

In a recent article, Bertolino et al. (2020) presented a horizon-scanning approach to invasive mammal species in Italy, contributing to ongoing EU and national efforts to assess and manage invasive species. Bertolino et al. 2020 then suggest a list of six priority species for policy-making, combining information from standard impact assessment methods (e.g. Environmental Impact Classification of Alien Taxa, EICAT; Blackburn et al. 2014) with additional considerations about manageability. Moving from risk assessment to management decisions is challenging, and the species prioritization by Bertolino et al. encounters some common pitfalls. Here, we highlight those pitfalls and suggest solutions based on best practices in conservation decision-making. We do not question the assessment of 'Impact' and 'Manageability'. Both are important and we commend Bertolino et al. for providing them. However, we caution against aggregating such different criteria into the 'Impact + Management' prioritization score (the overall ranking by Bertolino et al. 2020), when there is not a clear context or need for it, and the method used is arbitrary.

The first pitfall is that identifying priorities requires a clear decision context (who makes the decision, their objectives, their constraints; Keeney 1982; Game et al. 2013). The criteria used by Bertolino et al. (2020) are clear: impacts on biodiversity, economy, society and human health, and various criteria for manageability. The context for the assessment along these criteria is also clearly defined by the EU and the national requirement to "rank species to be subjected to risk assessment" (Bertolino et al. 2020 , p. 32). However, prioritization is necessary only when one must allocate limited resources, typically time and money. If these competing objectives are not defined, assessment cannot be meaningfully converted to prioritization (Kumschick et al. 2020).

Copyright Stefano Canessa et al. This is an open access article distributed under the terms of the Creative Commons Attribution License (CC BY 4.0), which permits unrestricted use, distribution, and reproduction in any medium, provided the original author and source are credited. 
To illustrate, imagine a situation where three species must be managed, ranked 1-2-3 from greatest to smallest impacts. If resources were available to manage only one, and they all cost the same, species 1 might be prioritized. If the cost of managing species 1 were equal to the cumulative cost for 2 and 3, one might decide to prevent damage from species 2 and 3 , instead of species 1 only. If resources were available for all three, prioritization would be unnecessary regardless of cost. To convert the risk assessment into a decision (prioritization), one must therefore define or assume a decision context, for example imagining the spatial, temporal, social scales where an action is feasible and has certain costs. This must be the case for manageability criteria expressed by Bertolino et al (2020). If the prioritization is to directly guide resource allocation, the context must be clearly defined and truly reflect all situations it might apply to.

On the other hand, management decisions will ultimately be case-specific (e.g. implying "different weights to the various aspects of the invasion process", Bertolino et al. 2020, p. 46). However, in this case prioritizing on a pre-conceived or assumed context is meaningless and possibly misleading, and it would be better to simply list the ranks for each criterion without aggregating them. For example, one may state that the assessment ranked " $S$. floridanus as the most impacting and easiest to manage alien mammal in Italy" (Bertolino et al. 2020, p. 44) and show the assessment criteria (essentially Table 2 in Bertolino et al. 2020, without columns 4-5), avoiding aggregate "prioritization" scores with their assumed value judgments and decision contexts (Game et al. 2013). Decision-makers can use this information to carry out their context-specific prioritization, using multi-criteria decision analysis if needed (Adem Esmail and Geneletti 2018).

Finally, if a clear context is missing but one still wishes to illustrate a "blueprint for similar prioritization initiatives" (Bertolino et al. 2020, p. 48), then this should be based on sound decision-analytic principles and ideally include illustrative examples of how priorities change with different objectives, management costs and resource constraints, such as annual budgets (Joseph et al. 2009; Dodd et al. 2017).

A second pitfall in the aggregation is arbitrariness. EICAT and similar approaches use qualitative scales, e.g. from Minimal Concern to Massive, based on rigorously defined criteria that, while inevitably arbitrary, remain internally consistent. However, this scale is essentially ordinal, not cardinal (i.e. represents order, not quantities): for example, category 4 (Major) is worse than category 2 (Minor), but not necessarily twice as bad. This does not change when converted to a numerical scale (1 to 5) as in Bertolino et al. 2020. Sums and multiplication of such scores still return numerical values that suggest objectivity, but remain fundamentally arbitrary and carry no biological meaning (Wolman 2006).

This problem is repeated by scoring manageability on the same 1-5 scale and multiplying it by the converted impact scores. A species with impact $I=1$ and manageability $M=5$ thus scores the same as one with $I=5$ and $M=1$, and half the score of a species with $I=5$ and $M=2$. But for management, species 1 and 2 might or might not be the same, and might or might not be twice as "priority" as species 3 . Because the original scales have no cardinal meaning, such aggregate scores can be unin- 
formative or misleading. In Bertolino et al. (2020), "Spread" and "Overall impacts" range up to 937.5 and 11718.75 , respectively. A decision-maker might conclude that an overall impact score of 1000 is half as bad as one of 2000, but there is no logical basis for this interpretation (Game et al. 2013). One should go back to the original verbal scale and ask themselves: what does it mean to multiply "Major impact" times "Low manageability"?

To reduce arbitrariness, one might express outcomes using natural scales, like probabilities for chance events (Game et al. 2013). For large-scale assessments, again it is better to resist the temptation to convert constructed scales to numbers and to aggregate scores. If an overall recommendation is needed, it might be equally arbitrary, but more transparent, to rank objectives or impact categories separately, then assign a subjective overall score using the same process, reporting value judgments clearly (Game et al. 2013).

A third pitfall is that uncertainty influences risk attitudes and priorities (Tulloch et al. 2015). For example, the expected impacts of species 1 might range from Minimal to Massive (1-5) and those for species B from Moderate to Major (3-4). Risk-averse managers might prioritize controlling species 1 which has the highest worst-case impacts, whereas risk-neutral managers might prioritize species 2 which has the highest "average" impacts (McCarthy 2014). We recommend making uncertainty explicit in any assessment, following best-practice for horizon-scanning and ICAT assessments (Roy et al. 2018; Volery et al. 2020). Quantifying uncertainty also improves expert performance in elicitations (Speirs-Bridge et al. 2010).

Invasive species are a severe and challenging threat to biodiversity, and rigorous risk assessments help manage them. Principles and methods from decision science can then complement those assessments, clarifying decision problems and reducing arbitrariness, to prioritize efforts and improve outcomes.

\section{References}

Adem Esmail B, Geneletti D (2018) Multi-criteria decision analysis for nature conservation: A review of 20 years of applications. Methods in Ecology and Evolution 9: 42-53. https:// doi.org/10.1111/2041-210X.12899

Bertolino S, Ancillotto L, Bartolommei P, Benassi G, Capizzi D, Gasperini S, Lucchesi M, Mori E, Scillitani L, Sozio G, Falaschi M, Ficetola GF, Cerri J, Genovesi P, Carnevali L, Loy A, Monaco A (2020) A framework for prioritising present and potentially invasive mammal species for a national list. NeoBiota 62: 31-54. https://doi.org/10.3897/neobiota.62.52934

Blackburn TM, Essl F, Evans T, Hulme PE, Jeschke JM, Kühn I, Kumschick S, Marková Z, Mrugała A, Nentwig W, Pergl J, Pyšek P, Rabitsch W, Ricciardi A, Richardson DM, Sendek A, Vilà M, Wilson JRU, Winter M, Genovesi P, Bacher S (2014) A unified classification of alien species based on the magnitude of their environmental Impacts. PLoS Biology 12: e1001850. https://doi.org/10.1371/journal.pbio.1001850 
Dodd AJ, Ainsworth N, Hauser CE, Burgman MA, McCarthy MA (2017) Prioritizing plant eradication targets by re-framing the project prioritization protocol (PPP) for use in biosecurity applications. Biological Invasions 19: 859-873. https://doi.org/10.1007/s10530016-1335-7

Game ET, Kareiva P, Possingham HP (2013) Six common mistakes in conservation priority setting. Conservation Biology 27: 480-485. https://doi.org/10.1111/cobi.12051

Joseph LN, Maloney RF, Possingham HP (2009) Optimal allocation of resources among threatened species: a project prioritization protocol. Conservation Biology 23: 328-338. https:// doi.org/10.1111/j.1523-1739.2008.01124.x

Keeney RL (1982) Decision analysis: an overview. Operations Research 30: 803-838. https:// doi.org/10.1287/opre.30.5.803

Kumschick S, Bacher S, Bertolino S, Blackburn TM, Evans T, Roy HE, Smith K (2020) Appropriate uses of EICAT protocol, data and classifications. NeoBiota 62: 193-212. https:// doi.org/10.3897/neobiota.62.51574

McCarthy MA (2014) Contending with uncertainty in conservation management decisions. Annals of the New York Academy of Sciences 1322: 77-91. https://doi.org/10.1111/ nyas. 12507

Roy HE, Rabitsch W, Scalera R, Stewart A, Gallardo B, Genovesi P, Essl F, Adriaens T, Bacher S, Booy O, Branquart E, Brunel S, Copp GH, Dean H, D’hondt B, Josefsson M, Kenis M, Kettunen M, Linnamagi M, Lucy F, Martinou A, Moore N, Nentwig W, Nieto A, Pergl J, Peyton J, Roques A, Schindler S, Schönrogge K, Solarz W, Stebbing PD, Trichkova T, Vanderhoeven S, van Valkenburg J, Zenetos A (2018) Developing a framework of minimum standards for the risk assessment of alien species. Journal of Applied Ecology 55: 526-538. https://doi.org/10.1111/1365-2664.13025

Speirs-Bridge A, Fidler F, McBride M, Flander L, Cumming G, Burgman MA (2010) Reducing overconfidence in the interval judgments of experts. Risk Analysis 30: 512-523. https:// doi.org/10.1111/j.1539-6924.2009.01337.x

Tulloch AIT, Maloney RF, Joseph LN, Bennett JR, Di Fonzo M, Probert WJ, O'connor SM, Densem JP, Possingham HP (2015) Effect of risk aversion on prioritizing conservation projects. Conservation Biology 29: 513-524. https://doi.org/10.1111/cobi.12386

Volery L, Blackburn TM, Bertolino S, Evans T, Genovesi P, Kumschick S, Roy HE, Smith KG, Bacher S (2020) Improving the Environmental Impact Classification for Alien Taxa (EICAT): a summary of revisions to the framework and guidelines. NeoBiota 62: 547-567. https://doi.org/10.3897/neobiota.62.52723

Wolman AG (2006) Measurement and meaningfulness in conservation science. Conservation Biology 20: 1626-163. https://doi.org/10.1111/j.1523-1739.2006.00531.x 